\title{
Contribution to the Optical Design of A Concentrator with Uniform Flux for Photovoltaic Panels
}

\author{
Stanislas Sanfo ${ }^{1}$, Abdoulaye Ouedraogo ${ }^{2, *}$ \\ ${ }^{1}$ Laboratory of Physics and Chemistry of the Environment (LPCE), Department of Physics, University of Ouagadougou, Ouagadougou, Burkina Faso \\ ${ }^{2}$ Laboratory of Thermic and Renewable Energies (LETRE), Department of Physics, University of Ouagadougou, Ouagadougou, Burkina Faso
}

Copyright (C) 2015 by authors, all rights reserved. Authors agree that this article remains permanently

open access under the terms of the Creative Commons Attribution License 4.0 International License

\begin{abstract}
In this investigation the aim is to broaden the space of optics for Low Concentrating Photovoltaic applications. The main target is to achieve the concentration of sunlight uniformly over a large photovoltaic receiver. To solve this problem we implemented a Monte-Carlo method and defined a new parameter in order to facilitate the analysis of irradiance distributions. The geometric parameters to consider in order to reach the optimization goals have been established by dichotomous search. Utilizing this numeric method, we analyzed the influence of several geometrical parameters on the behavior of hypothetical optics. With this procedure we obtained a probative configuration made of simple geometries, leading to an uniform irradiation distribution.
\end{abstract}

Keywords Concentrating Photovoltaic, Optical design, non imaging optics, Uniform illumination, ray tracing

\section{Introduction}

Nowadays, one of the core research in Photovoltaic technology is dedicated to the design of Concentrated Photovoltaic (CPV) [13]. The concept of CPV technology is to collect sunlight from a large area and to concentrate it on a smaller Photovoltaic receiver. Consequently, for the same quantity of electricity, the costs are reduced by using a concentrator of low cost in order to obtain an amount of energy comparable to the effect of several suns on expensive photovoltaic cells. Historically, this solution has taken a great consideration with the development of solar cells of high-efficiencies, like mono crystalline, having an efficiency of $17.5 \%$ to $22.5 \%$, or multi-jonction solar cells[8], [15]. The photovoltaic technology combining high-efficiency solar cells and concentrating optics have been widely designed and proposed [10], [15], [12].

Among CPV optical systems using reflection of the light instead of refraction, classical parabolic reflectors are widely used as well as more complex configurations, which may be made of a primary concentrator with a Secondary Optical Element (SOE). These secondary elements work on the ray bundles provided by the primary in order to improve the concentration factor, the angular acceptance, and often the light uniformity[16]. A number of different designs for these components can be found, like domed shapes, Compound Parabolic Concentrators (CPC), truncated pyramids or cones, depending on the requirements of every CPV manufacturer.

In Burkina Faso, our investigations are among the first undertaken in the Photovoltaic Concentration field. Throughout the country, the technology of polycrystalline or monocrystalline flat-plates are been under progressive considerations by the government in its rural electrification projects. In this context a sensitive reduction of the investment costs will be of a considerable help. So, the general problem we attempt to address is the coupling of a solar concentrator with a PV panel; assuming that the PV panel can be made of many row of solar cells connected in series. It is known that a CPV's electrical efficiency in this case is strongly related to the distribution of the irradiation. As a matter of fact, a non-uniform distribution over such arrays of cells leads to a limitation of the output current due to the less illuminated cell, so that the efficiency decreases significantly [5], [7], [1]. On the other hand, it is assumed that an optical configuration which favours low sensitivities to optical errors contributes to reducing the impacts of the precision of manufacturing modules and tracking system failures.

In this paper, we discuss the design of a solar concentrator with the particularities of providing uniform distribution of irradiation and reducing the sensitivity to some optical errors. Hence, in the following lines we will investigate numerically such a non imaging solar concentrator among optics combining simple geometries such as parabolic shapes. The investigations are conducted by studying the influences of geometric parameters in a dichotomous approach where the appropriate conditions are established by elimination.

\section{Methodology and tools}

\subsection{Modeling of radiative heat transfer}

To simulate optical systems, many softwares or development environments have been developed such as Mirval, Soltrace, Stral and Tonatiuh, [2], EDStar [17]. They commonly use the Monte Carlo ray-tracing to predict the concen- 
trated solar flux distribution incident on a receiver [3], [4], [9]. In this approach, solar radiation is assumed to be composed of discrete energy bundles and the computation consists of following their probable paths which are generated randomly from the solar source up to the optical components [17]. The Radiative exchanges can be expressed in Transfer Equation that can be solved through its integral formulation. The Monte Carlo method in this case is used as a numerical method to calculate explicitly these integrals.

To achieve our objective we develope a code to obtain a fast scheme which does not generate heavy data. The integral formulation of Monte Carlo method can be found in many works [11], [6], [17], it depends on the quantity calculated and the assumption formulated to address the problem. In our concern, we consider that the simulation of linear concentrators in 2D is a simple and complete approach to find out the aspect of the distribution of rays because there is an unique and main direction of concentration. Then, the problem is considered in the transverse plane (i.e. the contribution of rays are neglected in longitudinal direction). We assume that all optical surfaces are perfect and ideal and they work in specular reflection conditions. The receiver is divided in $N_{x}$ parts of elementary size $\delta S$. A giving point $P$ on the receiver is considered as an extend element $\delta S_{p}$ surrounding the point $P$.

Consequently, the integral formulation of the Monte Carlo algorithm has been written as follows:

$$
\begin{aligned}
\varphi(P)= & \int_{S_{1}^{+}} p_{X_{1}}\left(x_{1}\right) d x \int_{\Omega_{S}} p_{\Theta}\left(\omega_{S}\right) d \omega \cdots \\
& \times\left\{\mathcal{H}\left(P\left|\omega_{S}\right| x_{1}\right) \times \widehat{\omega}_{A}\right\}
\end{aligned}
$$

with:

$$
\begin{aligned}
& \mathcal{H}\left(P\left|\omega_{S}\right| x_{1}\right)=\left\{\begin{array}{l}
H\left(x_{0}^{\prime} \in S_{2}\right) \cdot \widehat{\omega_{\text {out }}} \\
+H\left(x_{0}^{\prime} \notin S_{2}\right) \times\left\{\begin{array}{l}
H\left(x_{0}^{\prime} \in S_{3}^{+}\right) \cdot \widehat{\omega_{\text {in }, 1}} \\
+H\left(x_{1}^{\prime} \in S_{1}^{+}\right) \cdots
\end{array}\right.
\end{array}\right. \\
& \times\left\{\begin{array}{l}
H\left(x_{2} \notin S_{2} \cup S_{3}\right) \cdot \widehat{\omega_{\text {out }}} \\
+H\left(x_{2} \in S_{3}^{-}\right) \cdot \widehat{\omega_{\text {out }}} \\
+H\left(x_{2} \in S_{2}^{+}\right) \cdots
\end{array}\right. \\
& \times \begin{cases}H\left(x_{3} \notin S_{3}^{+}\right) \cdot \widehat{\omega_{\text {out }}} & \\
+H\left(x_{3} \in S_{3}^{+}\right) & \times H\left(x_{3} \in \delta S_{p}\right) \cdot \widehat{\omega_{\text {in }, 2}}\end{cases}
\end{aligned}
$$

$\widehat{\omega_{\text {out }}}=0 ; \widehat{\omega_{i n, 1}}=0$ and $\widehat{\omega_{i n, 2}}=1$

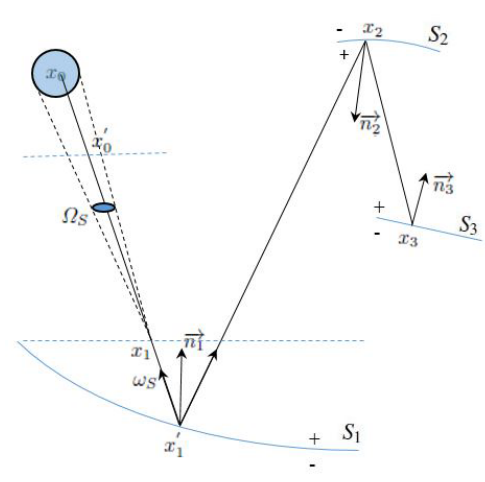

Figure 1. General diagram of ray path in a double stage optical system
In this investigation, Fig 1 shows how the path of a ray (from the sun to the receiver) is considered in a double stage reflection system. The optical loses are set by the heavy side function $\mathcal{H}\left(P\left|\omega_{S}\right| x_{1}\right)$ related to the number of rays lost in a given combination of interactions. The probability density functions ( $p d f s$ ) are defined by the simplified equations Eq. 3 and Eq. 4. The power brings by each ray is given in Eq. 5. The sensitivities to parameter are not taken into account in the expressions of $p d f s$. Instead, they are studied by running the program for each new given parameter.

$$
\begin{gathered}
p_{X_{1}}\left(x_{1}\right)=\frac{1}{S_{1}} \\
p_{\Omega_{S}}\left(\omega_{S}\right)=\frac{1}{\Omega_{s}} \\
\widehat{\omega}_{A}=\frac{I_{S}\left(\overrightarrow{\omega_{2}} \cdot \overrightarrow{n_{3}}\right)}{p_{\Omega_{S}}\left(\omega_{S}\right) p_{X_{1}}\left(x_{1}\right)}=I_{a}\left(\overrightarrow{\omega_{2}} \cdot \overrightarrow{n_{3}}\right) S_{1}
\end{gathered}
$$

The raytracing is detail in the following procedure:

1. Uniform generation of $N_{1}$ points $x_{1}$;

2. for each point $x_{1}$ an uniform generation of $N_{0}$ directions inside the angle of view of the sun;

3. calculate the impact points $x_{1}^{\prime}$ on the primary reflector;

4. calculate the normal $\overrightarrow{n_{1}}$ to the surface at $x_{1}^{\prime}$ applying the derivative of the equation of the primary;

5. calculate the new direction $\omega_{1}$ of the ray;

6. change the system of reference by rotation of angle $-\beta_{2}$ in order to be in the reference of the secondary;

7. calculate the impact point $x_{2}$ on the secondary (in the new system of reference);

8. calculate the normal $\overrightarrow{n_{2}}$ applying the derivative of the equation of the secondary (in the new system of reference);

9. calculate the direction $\omega_{2}$ of the ray (in the new system of reference);

10. determine the direction $\omega_{2}$ of the ray in the system of reference of the primary by rotation of angle $\beta_{2}$;

11. calculate the coordinates of the impact point $x_{3}$ on the receiver.

Sometime in the literature, instead of the brightness concentration ratio or the local concentration ratio, the uniformity of illumination over a surface is characterized by the optical concentration ratio; defined as the average irradiance integrated over the receiver area, divided by the insolation incident on the collector aperture [14]. Mathematically, this takes the form:

$$
C R_{0}=\frac{\frac{1}{S_{3}} \int \varphi(P) d S_{P}}{I_{a}}
$$


In the actual study, we define a new parameter $\left(\sigma_{C R_{0}}\right)$, called uniformity parameter. This parameter is a non dimensional quantity that holds a plurality of the positive gap between the ideal irradiance and the real irradiance over the receiver area, that is:

$$
\sigma_{C R_{0}}=\frac{1}{S_{3}} \int_{S_{3}}\left|\frac{\varphi_{\text {uniform }}-\varphi(P)}{\varphi_{\text {uniform }}}\right| d S_{P}
$$

The ideal irradiance is supposed to be constant over the receiver area and equal to:

$$
\varphi_{\text {uniform }}=\eta_{\text {opt }} \times C_{g} \times I_{a}
$$

We notice that all compensatory effect is avoided by the absolute value in Eq. 7; besides the optical efficiency of the concentrator is taken into account in Eq.8. The uniformity parameter is self explanatory; in a giving condition, if we tend toward a uniformed distribution of the irradiation, then the parameter $\sigma_{C R_{0}}$ will tend toward 0 , because the local flux will tend toward the stated ideal flux. Therefore, the uniformity of the irradiation profile can be inferred from the weight of $\sigma_{C R_{0}}$.

\section{Choice of convenient configuration}

First of all, we look for the solution to our specific problem among two stage optics with a primary concave parabolic reflector. The secondary mirror has to work on the prior converging ray bundles provided by the primary mirror in order to provide parallel emerging rays. So, we have the choice either the Cassegrainian type with a secondary convex parabolic reflector or the Gregorian type with a secondary concave parabolic reflector. The problem with classical configurations of these types is the fact that the secondary reflector casts shadow to the primary receiver so that the obtained spot on the receiver is separated in two parts. Instead of a complete trough parabola, we propose that a half trough parabola is considered as primary reflector.

Next we consider three values of $f-$ number, $f_{\# 1}=$ $0.6869,0.4330$, and 0.3570 obtained for specific aperture angles $\psi=40,60$, and 70 , if a complete through parabola of diameter $D=2 \mathrm{~m}$ is considered. We also defined non dimensional parameters as the ratio of the vertical separation between the primary mirror and the other components on the primary focal length:

$$
\begin{gathered}
\bar{h}_{2}=\frac{h_{2}}{f_{1}} \\
\bar{h}_{3}=\frac{h_{3}}{f_{1}}
\end{gathered}
$$

Fig. 2 shows the variances of the geometric concentration factor as a function of the Sub Reflector relative position $\bar{h}_{2}$, for different values of $f_{\# 1}$. One can see from the results in dotted lines obtained for the specific cases where $\bar{h}_{3}=0$, that there are optimum positions of the Sub Reflector for a giving $f_{\# 1}$. Considering a linear concentration, the maxima in Cassegrainian configuration are $C_{g}=4.43,5.54$ $\& 6.1$; while in Gregorian configuration the maxima are

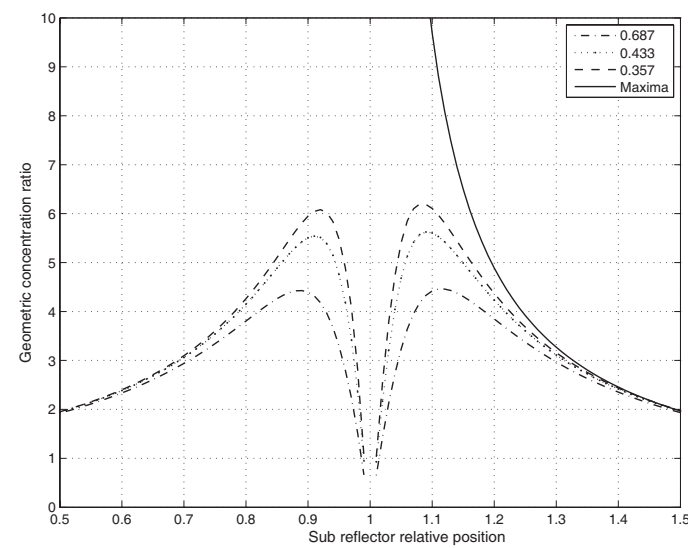

Figure 2. Variances of geometric concentration ratio with SR non dimensional position for different $f_{\# 1}$

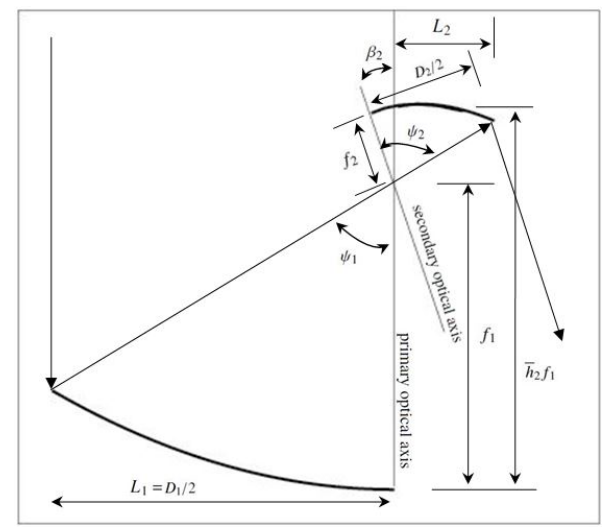

Figure 3. Off set Gregorian type with two half parabolic mirrors. The aligned axis is obtained when $\beta_{2}=0$.

$C_{g}=4.46,5.62 \& 6.20$; when $f_{\# 1}=0.6869,0.4330$, and 0.3570 , respectively.

The full line in Fig. 2 is obtained in Gregorian configuration, when the receiver is raised at the height $\bar{h}_{3}=1$. This translation increases the value of $C_{g}$, more significantly when $\bar{h}_{2}$ is closed to the value 1 . For the same values of $\bar{h}_{2}$ the obtained limits of the concentration are almost the same, whatever the value of $f_{\# 1}$, unless when $\bar{h}_{2}$ is decreasing.

Therefore, our choice is oriented toward the Gregorian configuration. If we consider the parameters as given in Fig. 3 and we apply them in the equation of parabola sets in polar coordinates, then the focus length of the secondary mirror and the ratio between the length of the two optics can be expressed as follows:

$$
\begin{gathered}
\left(1-\bar{h}_{2}\right) f_{1}=\frac{2 f_{2}}{1+\cos \left(\beta_{2}\right)} \\
\frac{L_{2}}{L_{1}}=\frac{4 f_{2} \sin \left(\psi_{1}\right)}{D_{1}\left(1+\cos \left(\psi_{2}\right)\right)}
\end{gathered}
$$

\section{Results of simulations}

The number of realizations of $N=1,000,000$ rays $(2,000$ positions $\times 500$ directions, taken uniformily) is assumed for the computation of radiative transfers by ray tracing. The value of $C_{g}$ in Eq.8 is calculated for every arrange- 
ment in the optical system and for every configuration of incident rays. For the study with aligned axis, no inclination is applied to the Sub Reflector as well as to the receiver. To investigate the off axis models, some simulations of inclined Sub Reflectors associated with the inclined receivers have been done in order to find out the potential changes. The non alignment of the two optical axis results from the inclination applied to the Sub Reflector; and, the inclination of the receiver is assumed because it is a necessary operation for reducing the cosine factor. To investigate the irradiation profile we consider that the mirrors have no physical limits; it means that the geometric factors determined by the "end effect", the blocking, the shading and the interception are not taken into account. These optical losses are studied apart.

\subsection{Outcomes with aligned axis}

\subsubsection{Geometric concentration factor}

Table 1. Size ratio and limit of concentration with SR position

\begin{tabular}{ccc}
\hline $\bar{h}_{2}$ & $C_{g, \text { limit }}$ & $\frac{L_{2}}{L_{1}}(\%)$ \\
\hline 1.1079 & $\mathbf{9}$ & 10.79 \\
1.0970 & $\mathbf{1 0}$ & 9.70 \\
1.0477 & $\mathbf{2 0}$ & 4.77 \\
1.0309 & $\mathbf{3 0}$ & 3.09 \\
1.0230 & $\mathbf{4 0}$ & 2.30 \\
1.0181 & $\mathbf{5 0}$ & 1.81 \\
1.0149 & $\mathbf{6 0}$ & 1.49 \\
1.0117 & $\mathbf{7 0}$ & 1.17 \\
\hline
\end{tabular}

Some data about the limit of concentration and the corresponding ratio between the optics according to the value of $\bar{h}_{2}$ are registered in Tab. 1 . Knowing that the sensitivities to optical errors are generally magnified for concentrators with $\frac{L_{2}}{L_{1}}$ low ratio, it should be suitable to envision the SR far from the focus point. We consider the percentage of $\frac{L_{2}}{L_{1}}$ arbitrarily between $4.77 \%$ and $20 \%$. If we refer to the results in Tab. 1 and the Fig. 2 it can be seen that the concentration factor we can reach is theoretically limited and set between 5 to 20

\subsubsection{Irradiation profiles and uniformity parameter}

Here, we investigate the effects of parameters $f_{\# 1}, \bar{h}_{2}$, and $\bar{h}_{3}$ on the probable profile of the irradiation distribution. Fig 4 shows the local concentration ratio (a) for $f_{\# 1}=0.6869$ (b) for $f_{\# 1}=0.3570$, over the normalized space occupied by the receiver, at different values of $\bar{h}_{2}$ and at different values of $\bar{h}_{3}$. It can be seen from the layouts that the profile is better when $\bar{h}_{3}=1$. In this specific case it seems that the local concentration ratio looses its constancy at the second extremity of the receiver as $f_{\# 1}$ increases.

If we analyze the causality between the irradiation profile and the uniformity parameter it can be pointed out that the weight of $\sigma_{C R_{0}}$ follows the distortion of the spot shape. This result is expected; $\sigma_{C R_{0}}$ decreases while $\bar{h}_{2}$ increases and increases while $f_{\# 1}$ decreases. As a reference, we arbitrarily consider the $\sigma_{C R_{0}} \leq 0.1$ values in order to have a good condition for expecting an uniformity. In this interval, it seems that at least $90 \%$ of the receiver aperture is uniformly covered, as in the example shown by Fig.4a for the case where $\bar{h}_{3}=1 \& \bar{h}_{2}=1.05$.

\subsubsection{Variance of uniformity parameter with physical errors}

Fig. 5 shows the variations of uniformity parameter according to the concentrator misalignment with the sun, in the case where $f_{\# 1}=0.6869$; the irradiation profiles at some remarkable points are illustrated in small squares.
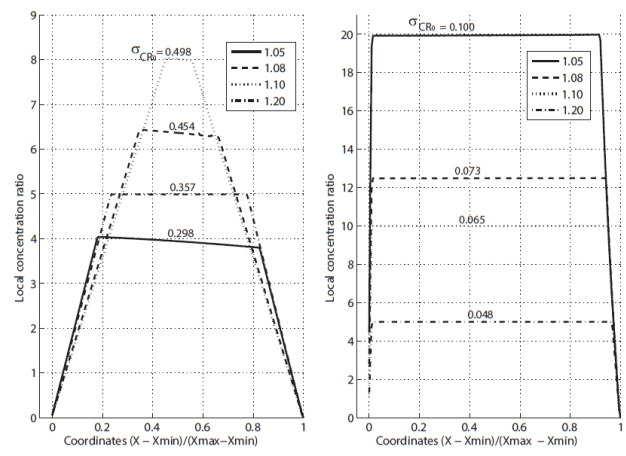

(a)
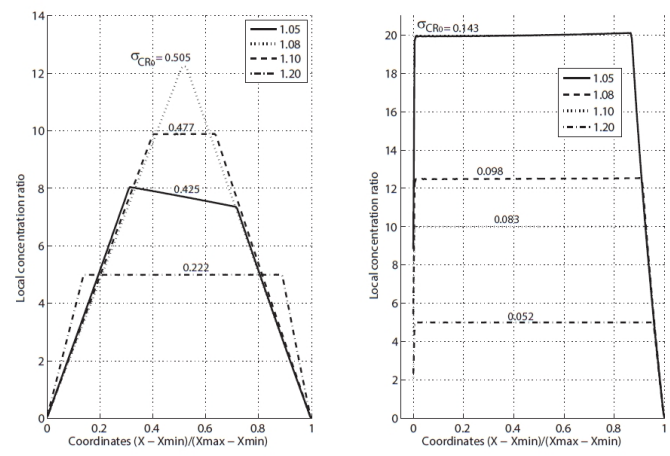

(b)

Figure 4. Probable aspects of the irradiation profiles a) $f_{\# 1}=0.6869$ with $\bar{h}_{3}=0$ on the left side and $\bar{h}_{3}=1$ on the right side; b) $f_{\# 1}=0.3570$ with $\bar{h}_{3}=0$ on the left side and $\bar{h}_{3}=1$ on the right side

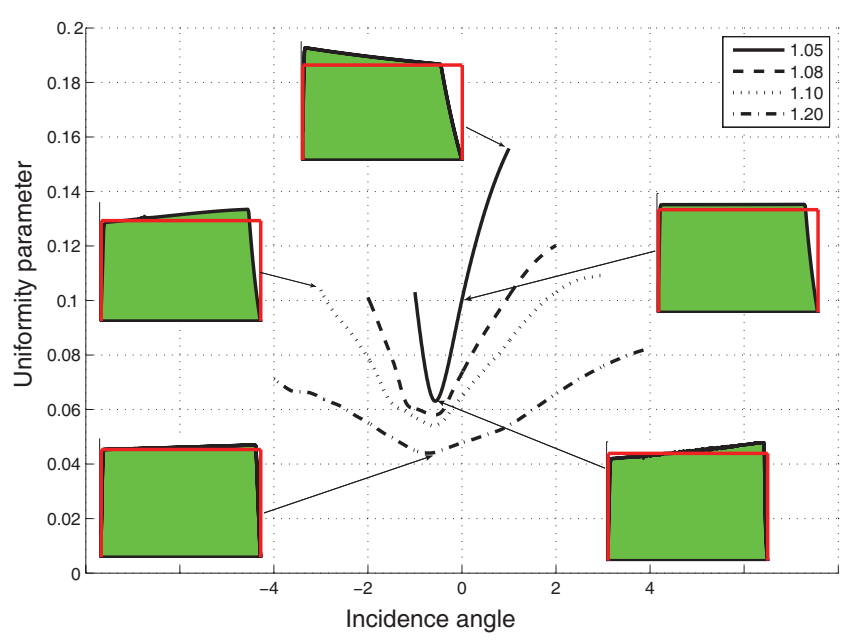

Figure 5. Variances of the parameter $\sigma_{C R_{0}}$ with incidence for different values $\bar{h}_{2}$.

One can observe that the uniformity parameter generates little improvements in negative deviation relatively to the perfect alignment, so that the best situation is obtained when $\theta \simeq-0.6$ degrees. On the basis of the requirement that $\sigma_{C R_{0}} \leq 0.1$, we notice a more significant flexibility in misalignment, around -0.6 degree, when $\bar{h}_{2}$ is high. Otherwise, 
with increasing incidence, the obtained profiles are progressively disturbed, even faster when $\bar{h}_{2}$ is low.

The same hierarchical organization of layouts with $\bar{h}_{2}$ values are observed when the calculations are done with a misplacement of the secondary optic in vertical direction, as well as in horizontal direction (see Fig. 6a \& Fig.6b).



(a)

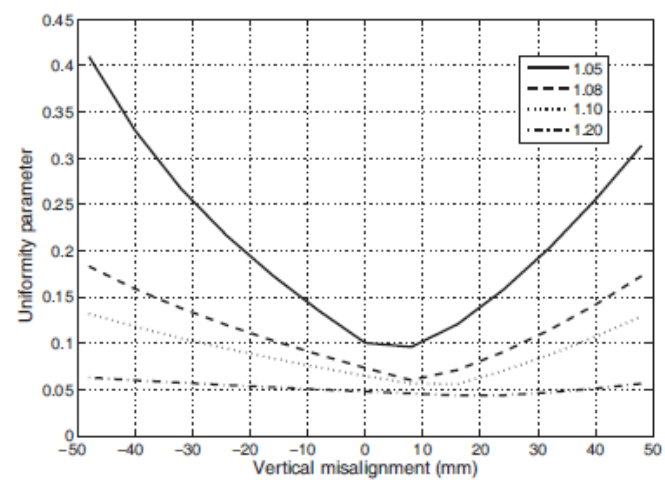

(b)

Figure 6. Effect of component misalignment on the uniformity parameter (a) the horizontal displacement of the SR ; (b) the vertical displacement of the SR.

\subsection{Optical losses}


Figure 7. Optical losses function of incidence angle

Here we analyzed the optical losses as a function of the angle of incidence, considering that the receiver is raised at the optimum height $\bar{h}_{3}=1$.
Fig. 7 shows the way optical losses vary with incidence angle. From these diagrams we can infer that each component, behaving independently, affects the transfer of the rays to the receiver. Considering a clockise rotation, for a negative increase of incidence angle, the rays do not suffer from any blockage. Rather, one observes a progressive increase in shading and overflowing due to interactions with the Sub Reflector or with the receiver. For a positive increase of incidence angle, the factor of shade is nonexistent while the value of the factor of blocking is remarkable. Immediately after the normal incidence, all the rays are blocked by the backside of the receiver.

In proportion to the remaining number of rays after a preceding interaction, the classification of the geometrical influences per degree of harmful effect is as follows : with negative incidence, the overflow on the receiver is more dominating, followed by the shade carried by the Sub Reflector; with positive incidence, there is the blocking effect by the receiver then the overflow from the Sub Reflector. The optical losses which result from the combination of all these influences increase very quickly. One can foresee that a considerable lost of the transmitting rays is perceived by the receiver, and make it difficult to reach a convenient acceptance angle. But it is possible to overcome this problem by envisioning a trend toward "off axis" models in order to move away the receiver from the primary axis.

\subsection{Getting toward off axis}

Fig.8a shows the variances of $\sigma_{C R_{0}}$ with respect to the Sub reflector inclination $\left(\beta_{2}\right)$, for given values of $\bar{h}_{2}$, while the receiver inclination is set to $\beta_{3}=0$ degree. The layouts show two phases in the variances of $\sigma_{C R_{0}}$, more noticeable when $\bar{h}_{2}$ is low: a decrease, follows by a raising. In $\beta_{2}$ studied value range we notice that $\sigma_{C R_{0}} \leq 0.1$. Fig. $8 \mathrm{~b}$ shows the variances of $\sigma_{C R_{0}}$ with respect to the receiver inclination, for given values of $\bar{h}_{2}$ and $\beta_{2}$. Here the layouts show linear correlations with positive slopes between the receiver inclination and the parameter $\sigma_{C R_{0}}$. It appears that $\sigma_{C R_{0}}$ increases faster with $\beta_{3}$, for example, in the case where $\bar{h}_{2}=1.05$ it is found that $\sigma_{C R_{0}}>0.1$ as soon as $\beta_{3}$ reaches 9 degrees.

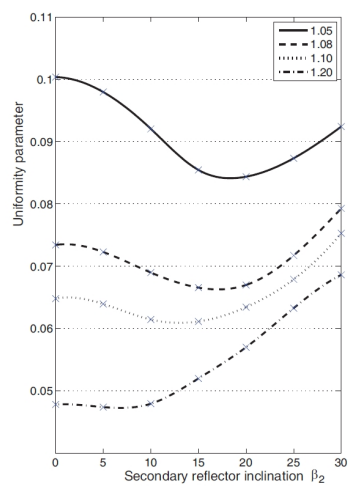

(a)

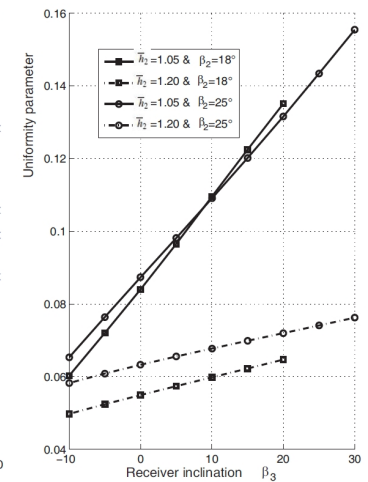

(b)
Figure 8. Variances of uniformity parameter with a) Sub Reflector inclination $b)$ receiver inclination for a given inclination of SR

One can approximate the cosine factor by $\cos \left(\beta_{2}-\beta_{3}\right)$. The results of the simulation show that this expression makes a good approximation of the cosine factor obtained numerically. So, the cosine factor is reducible by increasing the value of $\beta_{3}$ in a manner of making it to be close to the value of $\beta_{2}$. But, it can be inferred from Fig. $8 \mathrm{~b}$ that $\sigma_{C R_{0}}$ decreases 


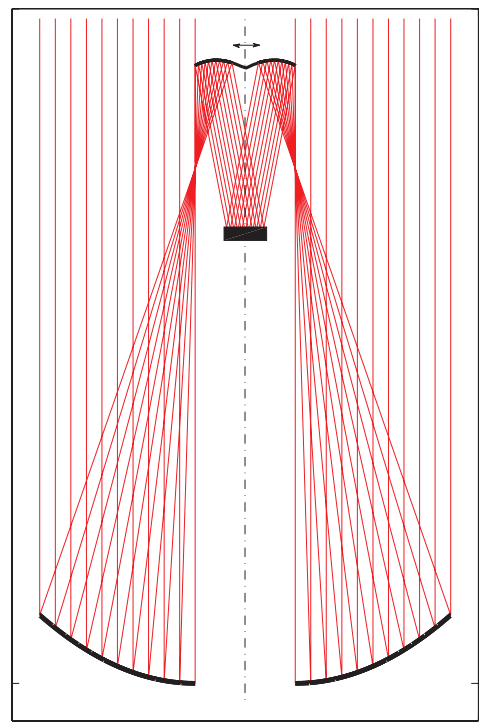

Figure 9. Retained configuration

only when the receiver is inclined in opposite direction.

Moreover, in considering additional results, it is found that for $\beta_{3}=0$ degree, the geometric concentration factor decreases with the increasing of the inclination of the SR because the spot size is enlarging; and for a fixed value of $\beta_{2}$ the concentration factor increases when $\beta_{3}$ tends toward $\beta_{2}$.

\section{Interpretation and discussion}

According to the previous results, it can be inferred that the concentration factor and the distribution of the irradiance can be greatly improved if the receiver is raised at a height close to the primary focus. The irradiance uniformity will show some improvements if the primary $f-$ number is high; or the separation between the secondary mirror and the receiver is also high. If the separation between the secondary mirror and the receiver is increased, the intercepting aperture of the secondary mirror will increase (the ratio between the two optics will increase); the sensitivity to physical errors will be notably reduced; but, the concentration factor will decrease slightly. If the design is considered with inclined emerging rays, it should be possible to move the receiver away in order to reduce the blocking problem and improve the acceptance angle. This operation does not damage the aspect of the irradiation profiles under 25 degrees of inclination. It should be possible to improve the irradiation profile by applying a positive inclination to the secondary mirror, or applying a negative inclination to the receiver. However, the outcomes of these operations will impact on the concentration factor and the intercepting factor; for both factors a slightly decrease will be more or less noticeable with the increase of the secondary inclination and a raising will be noticed with the decrease of the difference between the inclination of the secondary mirror and the inclination of the receiver.

Taking into account these conclusions we proposed the new optical configuration shown by Fig 9. The proposed non imaging optic is a Gregorian type made of two half primary concave mirrors and two Sub convex parabolic mirrors as- sembled to form a CPC secondary optical element. Referring to the design process, here the inclination of the secondary mirror is applied in order to allow the superposition of the spots given by two double stage concentrators arranged in symmetry. With this setup it is almost possible to double the concentration factor and save the level of the sensitivity to optical errors. The drawback is that the horizontal position of the receiver will lead to a decrease of the intercepting factor. A separation can be set between the two Sub Reflectors, in order to increase the acceptance angle by extending the possible race of rays over the Sub mirror. In this case, the extension curves have to be determined in a manner to keep the spot in the same borders.

Table 2 shows the improvements brought if we consider some examples of such configuration compares to classical configuration. The classical configuration adopts the aligned axis (i.e. $\beta_{2}=0$ degree), while the new configuration is applied with inclined axis such as $\beta_{2}=25$ degrees. In both cases $\beta_{3}=0$ degree. It is important to notice the possibility of adopting this configuration with mirrors of lower $f$ - number, due to the fact that the superposition of the two spots balances the irradiation profile, and improves it.

\section{Conclusion}

In this paper, the problem of designing a specific solar concentrator has been resolved in two steps: first of all, presupposed conform optics are established and secondly a dichotomous analysis of parametric influences is done in order to find out the suitable configurations. Analysing the optics using a Monte Carlo code we point out the influences of geometric parameters on some optical efficiency factors.

We show that the introduced parameter $\left(\sigma_{C R_{0}}\right)$ is suitable in order to easily compare the degree of the uniformity of the irradiance distributions provided by the numerous studied cases. If two stage optic system is envisioned, we show that classic configuration using simple parabolic shapes has the best concentration of 6.2 and the distribution of the irradiation is twisted. At the end of this study, the best trade-off we propose is to arrange half parabolic trough in a way to obtain the better optical configurations showed by Fig 9. With this configuration there is no need of a third optical component. If this configuration is adopted with a high $f-$ number, it is possible to improve the geometric concentration factor by almost 5 times, while the uniformity of the distribution of the irradiation is appreciable. With a low $f$-number the size of the system can be reduced; in such case, a geometric concentration factor of 18 can be reached with an appreciable distribution of the irradiation.

For application purpose, we plan that a linear concentrator with the new design will allow the optimization of the irradiance distribution and the geometric concentration factor. In so doing, an easy to build concentrator is envisioned to open a broad vista of applications for an efficient concentration of solar energy on photovoltaic panels. The rotation of the retained configuration about the symmetry axis will result in a variant of truncated parabolic dish combined to a 3D-CPC; with such concentrator one can foresee the perspectives of reaching a uniform distribution of irradiation and a considerable degree of concentration range between 67 to 1026 . 


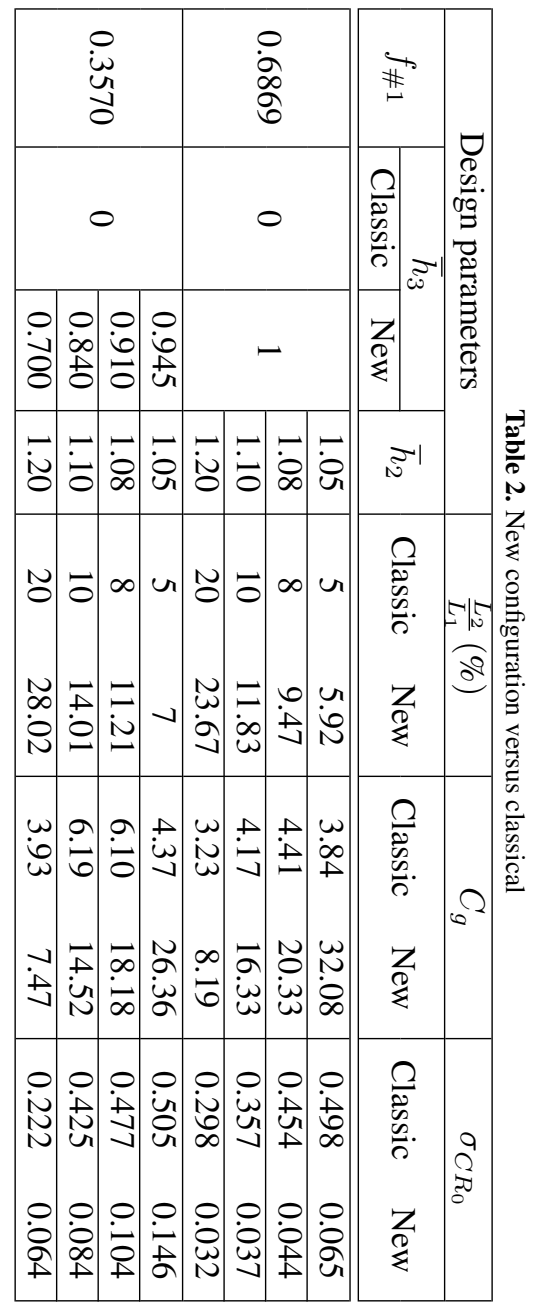

\section{Nomenclature}

\begin{tabular}{|c|c|}
\hline RS & Secondary reflectors \\
\hline $\mathbf{R P}$ & Primary reflectors \\
\hline $\mathbf{R C}$ & Receiver \\
\hline SOE & Secondary Optical Element \\
\hline index 0 & refers to a point on the sky or the sun \\
\hline index 1 & refers to an element from the primary \\
\hline index 2 & refers to an element from the secondary \\
\hline index 3 & refers to an element from the receiver \\
\hline$\theta$ & $\begin{array}{l}\text { angle of incidence at the primary } \\
\text { aperture }\end{array}$ \\
\hline$\beta$ & inclination of a component \\
\hline$\psi$ & parabolic mirror aperture angle \\
\hline$\omega$ & direction of an incoming ray \\
\hline$\Omega_{S}$ & sun angle of view \\
\hline $\mathcal{H}$ & heavy side function \\
\hline$H$ & $\begin{array}{l}\text { heavy side function for specific } \\
\text { optical loss }\end{array}$ \\
\hline$D$ & diameter of a parabola in meter \\
\hline$L$ & length of a component in meter \\
\hline$f$ & focus length in meter \\
\hline$S$ & optical surface \\
\hline$S^{+}$ & front side of an optical surface \\
\hline$S^{-}$ & back side of an optical surface \\
\hline$\delta S$ & element of surface over the receiver \\
\hline$x, x^{\prime}$ & variables, positions, interaction positions \\
\hline$h$ & height of a component \\
\hline $\bar{h}$ & algebraic value of the height $h$ \\
\hline$\vec{n}$ & normal vector to an optical surface \\
\hline$N$ & number of rays choosen for a simulation \\
\hline$p(x)$ & density probability function \\
\hline$\widehat{\omega}$ & weight \\
\hline$C_{g}$ & geometric concentration ratio \\
\hline$C R(P)$ & $\begin{array}{l}\text { local concentration ration at a giving } \\
\text { point } P\end{array}$ \\
\hline
\end{tabular}

\section{Acknowledgments}

We thank the FONRID (National Fond of Research and of Innovation for Development) for financing the publication of this article.

\section{REFERENCES}

[1] H. Baig, K. C. Heasman, and T. K. Mallick. Non-uniform illumination in concentrating solar cells. Renewable \& sustainable energy reviews, pages 5890-5909, 2012.

[2] G. BAUD, J. J. BEZIAN, M. E. HAFI, and G. OLALDE. Radiative heat transfer modelling in a concentrated solar energy bubbling fluidized bed. Journal of Physics, 2013.

[3] M. Carlini, C. Cattani, and A. O. Tucci. Optical Modelling of Square Solar Concentrator. IV:6785, 2011.

[4] C.-F. Chen, C.-H. Lin, H.-T. Jan, and Y.-L. Yang. Design of a solar concentrator combining paraboloidal and hyperbolic mirrors using ray tracing method. Optics Communications, 282(3):360-366, Feb 2009. 
[5] E.T.Franklin and J. Coventry. Effects of Highly Non-uniform Illumination Distribution on Electrical Performance of Solar Cells. Australian National University, 1983.

[6] O. FARGES, J.-J. BEZIAN, M. E. HAFI2, and H. BRU. Simulation of the annual performances of an installation with concentrated solar energy. 2013.

[7] I. Garcia, C. Algora, Rey-Stolle, and B. Galiana. Study of nonuniform light profiles on high concentration III-V solar cells using quasi-3D distributed models. E.T.S.I Telecomunication, 2008.

[8] S. Gite and P. Walke. A review of Photovoltaic cell with concentrating Collector. International Journal of Engineering Research \& Technology (IJERT), Vol. 2 Issue 3:2278-0181, March 2013.

[9] D. Groulx and B. Sponagle. RAY-TRACING ANALYSIS OF A TWO-STAGE SOLAR CONCENTRATOR Dominic Groulx, Benjamin Sponagle. Transactions of the Canadian Society for Mechanical Engineering, 34, No. 2(2):263-275, 2010.

[10] M. Khamooshi, H. Salati, F. Egelioglu, A. Hooshyar Faghiri, J. Tarabishi, and S. Babadi. A Review of Solar Photovoltaic Concentrators. International Journal of Photoenergy, 2014:1-17, 2014.

[11] J. D. la Torre. Calculation of sensitivities by the Monte-Carlo method, for the design of processes to concentrate solar energy. $\mathrm{PhD}$ thesis, Institut National Polytechnique of Toulouse University of Toulouse, 2011.

[12] A. Luque and S. Hegedus, editors. Handbook of Photovoltaic Science and Engineering. 2002.

[13] J. Nilsson. Optical Design and Characterization of Solar Concentrators for Photovoltaics. Master's thesis, Division of Energy and Building Design, Department of Architecture and Built Environment, Faculty of Engineering LTH, Lund University, 2005.

[14] R. M. Ochieng and F. N. Onyango. Some Techniques in Configurational Geometry as Applied to Solar Collectors and Concentrators. October, 2010.

[15] B. Parida, S. Iniyan, and R. Goic. A review of solar photovoltaic technologies. Renewable and Sustainable Energy Reviews, 15(3):1625- 1636, Apr 2011.

[16] W. R., J. C. Minano, and P. Benitez. Nonimaging Optics. 2005.

[17] F. Veynandt. Heliothermodynamic Cogeneration with linear Fresnel concentrator: modeling of the whole process. $\mathrm{PhD}$ thesis, Institut National Polytechnique of Toulouse University of Toulouse, 2001. 